\title{
Parasitosis intestinal en poblaciones urbana y rural en Sandia, Departamento de Puno, Perú
}

\author{
LUIS MARCOS*, VICENTE MACO*, ANGELICA TERASHIMA*, FRINE SAMALVIDES**, \\ ELBA MIRANDA** y EDUARDO GOTUZZO*.
}

\section{INTESTINAL PARASITOSES IN RURAL AND URBAN POPULATIONS IN SANDIA, DEPARTMENT OF PUNO, PERÚ}

The objective of the present,study, is to determine the rate of intestinal parasitoses in rural and urban populations of Sandia district, located at 2178 msnm., department of Puno, Perú. Fecal samples, 35 from rural (Group A) and 37 from urban (Group B) populations were collected (total 72). Four parasitological tests were used: Direct Microscopy, Kato Technique, Spontaneous Tube Sedimentation Technique and Modified Cup Baermann Technique. Global prevalences of intestinal parasitoses in both groups were high (A: $88.58 \%$; B: 67.57\%). Prevalence of intestinal parasitoses was: Ascaris lumbricoides (A: 51.42\%; B: 29.72\%, $p=0.06$ ), Entamoeba coli (A: 48.57\%; B: $18.91 \%, p=0.007)$, Trichuris trichiura (A: 42.85\%; B: 13.51\%, $p=0.005)$, Blastocystis hominis (A: 40\%; B: 32.42\%, $p=0.5$ ), Endolimax nana ( $A: 37.14 \%$; $B: 16.66 \%, p=0.04$ ), Giardia lamblia (A: $25.71 \%$; B: $13.51 \%, p=0.19$ ), Hymenolepis nana (A: 0\%; B: 5.4\%), Iodamoeba butschli (A: 2.85\%; B: 0\%), Entamoeba histolytica / E dispar (A: 2.85\%; B: 0\%), Strngyloides stercoralis (A: 2.85\%; B: 0\%) and Ancylostoma duodenale / Necator americanus (A: 0\%; B: 2.7\%). We conclude that intestinal parasitism is more frequent in rural than urban populations, is associated to poverty, poorer environmental sanitation and lack of hygienic services. Due to the high rates of parasitic infection we found, it is important to perform adequate parasitological tests to allow optimal diagnosis and opportune and effective treatment.

Key words: helminths, protozoos, prevalence, Survey. Sandia, Perú.

\section{INTRODUCCIÓN}

La parasitosis intestinal en el Perú tiene alta prevalencia y constituye un problema de salud pública ya que dentro de las diez principales causas de muerte se encuentran las enfermedades infecciosas intestinales: $7,7 \% .{ }^{1}$ Se menciona que uno de cada tres peruanos porta uno o más parásitos en el intestino. ${ }^{2}$ La distribución de la parasitosis intestinal se presenta según las regiones geográficas del país (costa, sierra y selva), diferentes estudios muestran un predominio de helmintos en la selva, y protozoarios en la costa y sierra. Asimismo, dentro de estas regiones existe variación de la infección parasitaria entre la población rural y urbana. . $^{3-14}$

La prevalencia e intensidad de la infección están asociadas a mayor riesgo de morbilidad y tienden a ser elevadas principalmente en la población en edad escolar. Las deficientes condiciones sanitarias (ambientales, de infraestructura y educación) predisponen a esta

* Instituto de Medicina Tropical Alexander von Humboldt (IMTAvH). Av. Honorio Delgado 430, Urb. Ingienería, San Marín de Porres. A.P. 4314 - Lima 100, Perú. E-mail: egh@upch.edu.pe

** Laboratorio de Parasitología. Universidad Peruana Cayetano Heredia (UPCH), Lima, Perú. 
población a un mayor riesgo de infección por helmintos y protozoarios lo cual repercute en el estado nutricional.

Una de las formas más comunes de adquirir la infección por helmintos en los niños es la geofagia, que ha sido asociada con un riesgo incrementado de reinfección con Ascaris lumbricoides y posiblemente con Trichuris trichiura. ${ }^{15}$

El objetivo del presente trabajo fue determinar la prevalencia de infección por helmintos y protozoarios en dos grupos (urbano y rural) en una zona poco estudiada (Sandia), mediante el empleo de métodos coproparasitológicos rutinarios y selectivos.

\section{MATERIAL Y MÉTODOS}

El presente estudio fue realizado durante el mes de Agosto del 2000 en la ciudad de Sandia perteneciente a la selva alta de la provincia de Sandia, departamento de Puno, la cual está ubicada a $2.178 \mathrm{msnm}, 14^{\circ} 14^{\prime} 50^{\prime \prime}$ latitud sur y $69^{\circ} 25^{\prime} 30^{\prime \prime}$ longitud oeste, con una superficie aproximada de $1.693 \mathrm{Km}^{2}$ y una población estimada de 11.509 habitantes. Se estudiaron dos poblaciones: rural, comunidad de Chijisilla, distrito de Sandia (Grupo A) y urbana, ciudad de Sandia (Grupo B).

Se realizó una Campaña de Detección de Enteroparásitos en ambos lugares y al inicio se explicó a los participantes los objetivos del estudio; en el caso de los niños se les comunicó a los padres o tutores, de quienes se obtuvo el consentimiento informado verbal. No se realizó entrevista epidemiológica.

A cada persona se le entregó un frasco de plástico $(200 \mathrm{cc})$ rotulado con su nombre, apellido y un número de identificación; se tomó una muestra de heces por persona. Las muestras fueron recogidas el mismo día y se las conservó en formol al $10 \%$, posteriormente fueron trasladadas al Laboratorio de Parasitología del IMTAvH-UPCH en Lima, donde fueron procesadas mediante 4 exámenes coproparasitológicos: Examen Directo (ED), Técnica de Kato (K), Técnica de Sedimentación Espontánea en tubo (TSET) y Técnica de Baermann modificado en copa (TBMC).

El análisis estadístico se realizó en el EpiInfo v. 6.4, 1996 (CDC Atlanta, USA), un valor de $\mathrm{p}<0,05$ fue considerado como el nivel crítico de significancia.

\section{RESULTADOS}

El medioambiente y las condiciones de las viviendas variaron en ambos grupos. Todas las viviendas del grupo A estaban construidas de adobe o esteras, con piso de tierra y alrededor existen reservorios de agua como ríos, arroyos, charcos y riachuelos de donde obtienen agua para el consumo y donde los niños juegan. La mayoría no contaban con servicios higiénicos adecuados, las deposiciones (heces) las realizaban a campo abierto, en silos o letrinas. Los niños no usaban calzado.

Por el contrario, las viviendas del grupo B estaban construidas de ladrillo, con piso de cemento, no existían reservorios naturales de agua en las proximidades y el consumo de agua era en general potable. La eliminación de excretas las realizaban en servicios higiénicos propios, sin embargo, algunos utilizaban letrinas o silos. Algunos niños no usaban calzado y jugaban en pistas asfaltadas a diferencia del grupo A.

En total se incluyeron a 72 individuos $(41,7 \%$ hombres y 58,3\% mujeres), no hubo diferencia significativa entre ambos sexos. $\left(X^{2}=1,34 ; \mathrm{p}=\right.$ $0,24)$. La edad fluctuó entre 1 y 53 años (media \pm DS $=14,84 \pm 11,4)$. La mayoría eran niños y adultos jóvenes, el $88,9 \%$ del total tenía menos de 25 años y el 48,6\% menos de 10 años.

En el grupo A se incluyeron a 35 individuos (entre 1 y 53 años) y en el grupo B a 37 individuos (entre 1 y 25 años).

La prevalencia de enteroparásitos varió entre los dos grupos. El 88,58\% del grupo A $(\mathrm{n}=35)$ y $67,57 \%$ del grupo $B(n=37)$ estaban infectados con uno o más parásitos $\left(X^{2}=4,59 ; \mathrm{p}=0,032\right)$.

La prevalencia de parásitos patógenos en el Grupo A fue la siguiente: A. lumbricoides $51,42 \%$, T. trichiura 42,85\%, Giardia lamblia $25,71 \%$, Entamoeba histolytica/Entamoeba dispar 2,85\%, Strngyloides stercoralis 2,85\%. La prevalencia de enteroparásitos no patógenos fue la siguiente: Entamoeba coli $(48,57 \%)$, Blastocystis hominis (40\%), Endolimax nana (37,14\%), e Iodamoeba butschlii $(2,85 \%)$.

La prevalencia de parásitos patógenos en el Grupo B fue la siguiente: A. lumbricoides $29,72 \%$, G. lamblia 13,51\%, T. trichiura $13,51 \%$, H. nana 5,4\% y Ancylostoma duodenale/Necator americanus $2,7 \%$. La prevalencia de enteroparásitos no patógenos fue: B. hominis $32,42 \%$, E. coli $18,91 \%$, E. nana $16,66 \%$. (Tablas 1 y 2 ). 
La diferencia de prevalencias de trichiurosis entre ambos grupos fue la única que mostró diferencia significativa $\left(X^{2}=7,72 ; p=0,005\right)$.

En el grupo A se encontró un caso de $S$. stercoralis. En el grupo B se encontraron dos casos de $H$. nana y uno de uncinarias.

Por otro lado, la mayor prevalencia dentro de los protozoarios correspondió a B. hominis $32,42 \%$ en el grupo B; y E. coli $48,57 \%$ en el grupo A. En ambos grupos predominaron los protozoarios sobre los helmintos.

La frecuencia de multiparasitismo en ambos grupos se muestra en la Tabla 3. Dentro de las asociaciones parasitarias más frecuentes se encuentran: E.coli y E.nana $(19,44 \%)$, B. hominis y E. nana $(16,66 \%)$, B. hominis y E. coli (16,66\%), T. trichiura y A. lumbricoides (15,27\%), A. lumbricoides y B. hominis $(13,88 \%)$, B. hominis, E.coli y E. nana (12,5\%), G. lamblia y E. coli $(11,11 \%)$, G. lamblia y B. hominis $(11,11 \%), T$. trichiura y B. hominis $(9,72 \%), T$. trichiura, A. lumbricoides y B. hominis (6,94\%).

La Técnica de Sedimentación espontánea en tubo (TSET) mostró un mayor rendimiento tanto para el diagnóstico de helmintos como de protozoarios en comparación con el Examen Directo (ED) y la Técnica de Kato (K). Para el diagnóstico de A. lumbricoides (AL) y $T$. trichiura (TT) la TSET mostró diferencia significativa en comparación con el examen directo (AL: $X^{2}=6,41, \mathrm{p}=0,01$; TT: $X^{2}=17,38$, $\mathrm{p}=0,000)$.

Tabla 1. Prevalencia de parásitos patógenos en los grupos A (población rural) y B (población urbana)

\begin{tabular}{lrcr}
\hline $\begin{array}{l}\text { Parásitos } \\
\text { patógenos }\end{array}$ & $\begin{array}{c}\text { Población } \\
\text { rural } \\
\text { (Grupo A, n=35) } \\
\text { Prevalencia \% }\end{array}$ & $\begin{array}{c}\text { Población } \\
\text { urbana } \\
\text { (Grupo B, n=37) }\end{array}$ \\
& & & $\begin{array}{c}\text { (Prevalencia \%) } \\
\text { p }\end{array}$ \\
\hline $\begin{array}{l}\text { Ascaris lumbricoides } \\
\text { Trichuris trichiura }\end{array}$ & 51,42 & 29,72 & $\mathrm{NS}^{\alpha}$ \\
$\begin{array}{l}\text { Giardia lamblia } \\
\text { Hymenolepis nana }\end{array}$ & 25,71 & 13,51 & $0,005^{\beta}$ \\
$\begin{array}{l}\text { Entamoeba. histolytica / } \\
\text { Entamoeba. dispar }\end{array}$ & 2,85 & 0,0 & $\mathrm{NS}^{\chi}$ \\
$\begin{array}{l}\text { Ancylostoma. duodenale/ } \\
\text { Necator. Americanus }\end{array}$ & 0,0 & 2,7 & \\
Strongyloides stercoralis & 2,85 & 0,0 & \\
\hline
\end{tabular}

${ }^{\alpha} \mathrm{X}^{2}=3,52 ;{ }^{\beta} \mathrm{X}^{2}=7,72 ; \chi \mathrm{X}^{2}=1,71$

$\mathrm{NS}=$ No significativo
Tabla 2. Prevalencia de parásitos no patógenos en los grupos A (población rural) y B (población urbana)

\begin{tabular}{lccc}
\hline $\begin{array}{l}\text { Parásitos no } \\
\text { patógenos }\end{array}$ & $\begin{array}{c}\text { Población } \\
\text { rural } \\
\text { (Grupo A, n=35) } \\
\text { Prevalencia \% }\end{array}$ & $\begin{array}{c}\text { Población } \\
\text { urbana } \\
(\text { Grupo B, n=37) } \\
\text { (Prevalencia \%) } \\
\text { Pr }\end{array}$ \\
\hline Entamoeba coli & 48,57 & 18,91 & $0,007^{\phi}$ \\
Blastocystis hominis & 40,00 & 32,42 & $\mathrm{NS}^{\gamma}$ \\
Endolimax nana & 37,14 & 16,66 & $0,044^{\mu}$ \\
Iodamoeba butschlii & 2,85 & 0,0 & \\
\hline
\end{tabular}

${ }^{\phi} \mathrm{X}^{2}=7,12 ;{ }^{\gamma} \mathrm{X}^{2}=0,45 ;{ }^{\mu} \mathrm{X}^{2}=4,05$

$\mathrm{NS}=$ No significativo

Tabla 3. Frecuencia de multiparasitosis en las poblaciones rural (Grupo A) y urbana (Grupo B)

\begin{tabular}{lrcr}
\hline Multiparasitos & $\begin{array}{c}\text { Población } \\
\text { rural } \\
\text { (Grupo A, n=35) } \\
\text { Prevalencia \% }\end{array}$ & $\begin{array}{c}\text { Población } \\
\text { urbana } \\
(\text { Grupo B, n=37) } \\
(\text { Prevalencia \%) } \\
\mathbf{p}\end{array}$ \\
\hline Monoparasitosis & 17,14 & 29,72 & $\mathrm{NS}^{\eta}$ \\
Biparasitosis & 22,85 & 16,66 & $\mathrm{NS}^{\theta}$ \\
Triparasitosis & 20,0 & 10,81 & $\mathrm{NS}^{\sigma}$ \\
Tetraparasitosis & 14,28 & 10,81 & $\mathrm{NS}^{\psi}$ \\
Pentaparasitosis & 11,42 & 0,0 & \\
Hexaparasitosis & 2,85 & 0,0 & \\
\hline
\end{tabular}

${ }^{\eta} \mathrm{X}^{2}=1,58 ;{ }^{\theta} \mathrm{X}^{2}=0,51 ;{ }^{\sigma} \mathrm{X}^{2}=1,17 ;{ }^{\psi} \mathrm{X}^{2}=0,01$

$\mathrm{NS}=$ No significativo

\section{DISCUSIÓN}

Aunque el rango de edades es bastante amplio, aproximadamente el $90 \%$ eran niños, adolescentes y adultos jóvenes entre 1 y 25 años. Este estudio está orientado a analizar y discutir los resultados enfocándonos en la población joven.

La población urbana de Sandia tiene una menor prevalencia de enteroparásitos que la comunidad rural de Chijisilla. Sin embargo, ambas poblaciones presentan altas tasas de infección intestinal parasitaria aunque la zona urbana a diferencia de la rural sí cuenta con servicios sanitarios básicos, lo cual estaría asociado a la carencia de normas de higiene básicas y medidas preventivas elementales., ${ }^{3,9,12,13}$ 
Las poblaciones que viven unas cerca de otras pueden tener diferentes factores de riesgo para infectarse por enteroparásitos, especialmente si sus estándares socioeconómicos y condiciones de vida son diferentes. Por lo tanto, para un adecuado control es importante la realización de estudios epidemiológicos que sean capaces de detectar estas diferencias. Nuestro estudio presenta ciertas limitaciones, inherentes a la representación del tamaño muestral, selección de los casos, ausencia de entrevista epidemiológica y el diagnóstico realizado con muestras preservadas en formol que podrían haber afectado el diagnóstico de $S$. stercoralis o B. coli, los que se diagnostican con muestras frescas con la Técnica de Baermann modificado en copa (TBMC).

Este estudio fue realizado con la finalidad de describir, observar y reportar una zona remota y aislada de nuestra sociedad, donde no se han realizado estudios previos sobre parasitosis intestinal con más de una técnica diagnóstica y no se conocen con certeza los índices de infección parasitaria. Consideramos que este estudio provee una base para trabajos prospectivos. Debe resaltarse las técnicas de diagnóstico parasitológicas sencillas, económicas y eficaces, empleadas en este estudio, capaces de determinar con precisión y certeza los parásitos intestinales más frecuentes de una población de escasos recursos económicos. Conociendo las prevalencias se podrían ejecutar programas de control y prevención que evitarían el serio daño clínico que producen en el ser humano, especialmente en los niños.

La prevalencia de $S$. stercoralis fue del $2,85 \%$ en la comunidad rural de Chijisilla mientras que en la población urbana fue $0 \%$. La existencia de esta parasitosis en la zona rural se debe a que los pobladores (especialmente los niños) realizan sus labores rutinarias descalzos, exponiéndose permanentemente al medio ambiente selvático, donde las condiciones climáticas favorecen el desarrollo del ciclo de vida libre del parásito, como consecuencia la exposición es mayor en la zona rural que en la urbana. $^{10}$

Cabe señalarse que el Hospital de Apoyo de Sandia a través del Ministerio de Salud realiza campañas de desparasitación cada seis meses en la población escolar, mediante el empleo del Albendazol (antihelmíntico para A. lumbricoides $\mathrm{y}$ uncinarias, pero de efectividad moderada para trichiurosis); ${ }^{16}$ sin embargo, en este estudio observamos que las tasas de prevalencia de parasitosis son altas y que sería necesario una terapia farmacológica selectiva además de implementar medidas educativas de control y prevención de enfermedades parasitarias intestinales.

Es necesario un estudio de cohorte longitudinal que permita determinar la eficacia del tratamiento masivo en estas poblaciones.

Dentro de los helmintos el más prevalente en ambos grupos fue A. lumbricoides, confirmando ser la helmintiasis más frecuente en nuestro país. ${ }^{37,12,17}$ En segundo lugar se encuentra $T$. trichiura con altos porcentajes mostrando diferencia significativa entre ambos grupos $\left(X^{2}=\right.$ $7,72, p=0,005)$. Se conoce que estos parásitos están estrechamente asociados a la pobreza, desnutrición y en especial a malos hábitos higiénicos, dietéticos y culturales, que involucran en particular a la mayoría de las comunidades aborígenes que habitan la región, entre ellos los más afectados son los niños. Como se mencionó anteriormente la población escolar no cuenta con tratamiento antiparasitario adecuado, pues el empleado por el programa del Ministerio de Salud no tiene cobertura para todos los parásitos intestinales. Sugerimos que deben emplearse programas de control y prevención a largo plazo que permitan reducir el grado de exposición a las formas infectantes de los parásitos. Por otro lado, la solución inmediata la sugiere la propia Organización Mundial de la Salud (OMS), cuando propone a todos los gobiernos la desparasitación masiva de todos los grupos de riesgo, mediante la administración de monodosis de benzimidazoles una vez cada 6 meses. Esta propuesta, que aparentemente había fracasado en intentos anteriores, se ve hoy reivindicada cuando se demuestra que, si bien las posibilidades de infección son enormes, estas serán mucho menores si se emplea como criterio de monitoreo a la carga parasitaria, sin cambiar ninguno de los hábitos de los niños de riesgo, por el solo hecho de eliminarse cada vez menos parásitos, la contaminación sería menor. Progresivamente, este hecho derivará necesariamente en una disminución de la transmisión parasitaria, sin embargo, no resolverá los graves problemas sociales ya mencionados.

Dentro de los objetivos propuestos observamos que la Técnica de Sedimentación Espontánea en tubo (TSET) fue la de mejor rendimiento para el diagnóstico de enteroparásitos que el examen 
directo como lo describen también otros autores. ${ }^{18,19}$ Además, la TSET por su sencillez de ejecución y de procesamiento, su bajo costo y alto rendimiento, debería ser aplicado a gran escala lo que permitiría conocer con mayor precisión los índices de infección por enteroparásitos.

Este estudio concluye que la parasitosis es más frecuente en la población rural que en la urbana debido a factores socioeconómicos, menor saneamiento ambiental y ausencia de servicios higiénicos adecuados. Debido a las altas cifras de infección, deberían implementarse programas de control y prevención de enteroparásitos a corto y largo plazo, así como realizar exámenes coproparasitológicos adecuados a fin de obtener un diagnóstico óptimo y así brindar un tratamiento eficaz y oportuno que permita brindar una mejor calidad de vida a los pobladores.

\section{RESUMEN}

El objetivo del presente estudio de tipo descriptivo y de corte transversal, es determinar la tasa de enteroparasitismo en las poblaciones rural y urbana del distrito de Sandia, localizado a 2.178 msnm, departamento de Puno, Perú. Fueron recolectadas un total de 72 muestras de heces, 35 de la población rural (Grupo A) y 37, urbana (Grupo B). Se realizaron cuatro exámenes parasitológicos: Examen Directo, Técnica de Kato, Técnica de Sedimentación Espontánea en Tubo y Técnica de Baermann modificado en copa. La prevalencia global de parasitismo intestinal fue alta en ambos grupos (A: 88,58\%; B: $67,57 \%)$. La prevalencia de parásitos intestinales fue: A. lumbricoides (A: $51,42 \%$; B: 29,72\%, p =0,06), E. coli (A: 48,57\%; B: $18,91 \%$, $\mathrm{p}=0,007)$, T. trichiura $(\mathrm{A}: 42,85 \% ; \mathrm{B}: 13,51 \%$, $\mathrm{p}=0,005)$, B. hominis $(\mathrm{A}: 40 \% ; \mathrm{B}: 32,42 \%$, $\mathrm{p}=0,5)$, E. nana $(\mathrm{A}: 37,14 \% ; \mathrm{B}: 16,66 \%$, $\mathrm{p}=0,04)$, G. lamblia (A: 25,71\%; B: 13,51\%, $\mathrm{p}=0,19), H$. nana (A: 0\%; B: 5,4\%), I. butschli (A: 2,85\%; B: 0\%), E. histolytica / E. dispar (A: 2,85\%; B: 0\%), S. stercoralis (A: 2,85\%; B: $0 \%$ ) y A. duodenale/N. americanus (A: 0\%; B: $2,7 \%$ ). Este estudio concluye que la parasitosis es más frecuente en la población rural que en la urbana, asociado a la pobreza, escaso saneamiento ambiental y falta de servicios higiénicos. Debido a estas altas cifras de infección parasitaria, es importante realizar exámenes copropara- sitológicos adecuados con el fin de hacer un diagnóstico óptimo que permita brindar un tratamiento oportuno y eficaz.

\section{REFERENCIAS}

1.- ORGANIZACIÓN PANAMERICANA DE LA SALUD. Oficina Sanitaria Panamericana, Oficina Regional de la OMS. La Salud en las Américas ED. Washington DC; Publicación Científica, Vol 2 No 569,1998

2.- NÁQUIRA C. Parasitosis II: Diagnóstico y Tratamiento de las Enteroparasitosis. La Revista Médica 1997; 3 (18-19): 18-26.

3.- AZAÑERO R, BARRANTES C, BAMBAREN C, REBAZA C. Características de los enteroparásitos en escolares de Pango-Junín (Perú). III Congreso Peruano de Parasitología, 5, 1997.

4.- CÁRDENAS F, MARTÍNEZ E. Parasitismo intestinal en escolares de nivel primario de la localidad de EspinarCusco. III Congreso Peruano de Parasitología, 10, 1996.

5.- CONCHA R, HUIZA A, ESPINOZA Y et al. Estudio parasitológico en escolares de cinco centros educativos del distrito de Juan Guerra, provincia de Tarapoto, departamento de San Martín. III Congreso Peruano de Parasitología, 10, 1996.

6.- CORNEJO D A, GONZÁLES D, DIEGUES J. Estudio sobre Parasitismo intestinal en el Personal Técnico, Administrativo y Auxiliar de los Hospitales de Iquitos. Arch Per Patol Clin1948; 2: 123-30.

7.- DELGADO A. La helmintiasis intestinal en el distrito de Satipo. Arch Per Patol Clin 1995; 19: 130-1.

8.- LUMBRERAS H, CANTELLA R, BURGA M, GUERRA H. Estudio coproparasitológico en la colonia alemana del Pozuzo. Primer Congreso Nacional de Microbiología y Parasitología, Arequipa, 9, 1964.

9.- NÁQUIRA F, CÓRDOVA E, NÁQUIRA C. Contribución al estudio del parasitismo I. Observaciones preliminares en algunas localidades del Sur del Perú. Arch Per Patol Clin 1962; 16: 41.

10.- RODRÍGUEZ H. MARTÍNEZ E. Prevalencia de helmintos intestinales en escolares del nivel primario de Quillabamba-Cusco. III Congreso Peruano de Parasitología, 10, 1997.

11.- ROZAS M, MENDOZA Y, CABALLERO M, UGARTE N. Prevalencia de parasitosis por nematodos en niños de 4-14 años de las comunidades de Pacor y Vilcabamba-Distrito de Cacay-Provincia de Paucartambo-Departamento del Cusco. III Congreso Peruano de Parasitología, 7, 1997.

12.- SCOLARI C, TORTI C, BELTRAME A et al. Prevalence and distribution of soil-transmitted helminth (STH) infections in urban and indigenous schoolchildren in Ortiguera, State of Paraná, Brasil: implications for control. Tropical Medicine and International Health 2000; 5, 4: 302-7.

13.- VALDIVIA L, MONTESINOS J, NÁQUIRA F, CÓRDOVA E. El Parasitismo Intestinal en Quillabamba, Provincia de la Convención, Departamento del Cuzco. Boletín Peruano de Parasitología 1979; 1: 2-14.

14.- SISNIEGAS W, MARTÍNEZ E, LIU M. Parasitismo 
intestinal en la población escolar de la localidad de Chala, Caravelli-Arequipa. III Congreso Peruano de Parasitología, 13, 1997.

15.- GEISSLER P, MWANIKI D, THIONG'O F, FRIIS H. Geophagy as a risk factor for geohelminth infections: a longitudinal study of Kenyan primary schoolchildren. Transactions of the Royal Society of Tropical Medicine and Hygiene 1998; 92: 7-11.

16.- COLAN E, PAWLIKOSKY W, ACHAVAL A. Albendazol en el tratamiento de helmintiasis intestinales en niñas de Iquitos. Revista de Gastroenterología del Perú 1984; 4: 148-51.

17.- HALL A, ANWAR K, TOMKINS A, RAHMAN L. The distribution of Ascaris lumbricoides in human hosts: a study of 1765 people in Bangladesh. Transactions of the Royal Society of Tropical Medicine and Hygiene 1999; 93: 503-10.
18.- SALAS R, RODRÍGUEZ J. Evaluación de la técnica de sedimentación espontánea en el diagnóstico de enteroparásitos. III Congreso Peruano de Parasitología, 9, 1997.

19.- VERA L, TELLO R, TERASHIMA A, ALVAREZ H. Evaluación en campo de la técnica de sedimentación espontánea para el diagnóstico de enteroparasitosis. Revista Médica Herediana 1996; 7: 50-1.

Agradecimientos: al Dr. Félix Choque Mamani, Director de la REDESS Sandia, Hospital de Apoyo de Sandia, por brindarnos su apoyo para la realización de la Campaña de Detección de Enteroparásitos. Al Dr. Humberto Guerra, Jefe del Departamento de Microbiología del Instituto de Medicina Tropical Alexander von Humboldt y profesor principal de la Universidad Peruana Cayetano Heredia, por su colaboración en la revisión del artículo.

\section{CONGRESO LATINOAMERICANO} DE PARASOTOLOGÍA

\section{Temas Centrales}

- Enfermedad de Chagas

- Malaria

- Leishmaniosis

- Zoonosis parasitaria

- Parasitosis intestinales

\section{Simposium}

- Chagas transfusional

- Toxoplasmosis

- Complejo Teniosis Cisticercosis

- Hidatidosis

- Fasciolosis

- Parasitos emergentes y reemergentes

- Parasitologías en pre y post grado

Informaciones:

Casilla: 446 La Paz - Bolivia

Fax (591-2) 2227184

Email: reangles@ceibo.entelnet.bo

Tel: 2229275 - 2227184 - 2422044 